\title{
Análisis del uso y complicaciones del acceso vascular periférico en la técnica del recambio plasmático terapéutico mediante centrifugación
}

\author{
Yaiza Martínez-Delgadoㄹ, Guillermo Pedreira-Robles ${ }^{1}$, Ana Vasco-Gómez ${ }^{1}$, Cristina Herrera-Morales ${ }^{1}$, Sònia \\ Prochazka-Enrich $^{1}$, Ernestina Junyent-Iglesias ${ }^{1}$
}

${ }^{1}$ Servicio de Nefrología. Parc de Salut Mar. Barcelona. España

\begin{abstract}
Como citar este artículo:
Martínez-Delgado Y, Pedreira-Robles G, Vasco-Gómez A, Herrera-Morales C, Prochazka-Enrich S, Junyent-Iglesias $\mathrm{E}$. Análisis del uso y complicaciones del acceso vascular periferico en la técnica del recambio plasmático terapéutico mediante centrifugación. Enferm Nefrol. 2019 Jul-Sep;22(3):323-8
\end{abstract}

\section{Resumen}

Introducción: El recambio plasmático terapéutico es una técnica extracorpórea de depuración sanguínea. EI recambio plasmático terapéutico mediante el método de centrifugación (plasmacentrifugación) requiere flujos de $50 \mathrm{ml} / \mathrm{min}$ lo que permite realizar el intercambio con accesos vasculares periféricos. El principal objetivo de este estudio fué el de analizar el uso y las complicaciones del acceso vascular periférico en las sesiones de plasmacentrifugación.

Material y Método: Estudio observacional descriptivo longitudinal sobre el análisis de complicaciones del acceso vascular periférico de las sesiones de plasmacentrifugación realizadas entre enero de 2017 y marzo de 2018.

Resultados: Inclusión de un total de 93 sesiones de plasmacentrifugación, estas sesiones fueron llevadas a cabo en 9 pacientes con una mediana de 7 [P25:6; $P 75: 10,5]$ sesiones por paciente. El $66,66 \%(n=6)$ fueron mujeres. En el $89,24 \%(n=83)$ de las sesiones se usó la centrifugación y en el $10,75 \%(n=10)$ la fotoaféresis.

La etiología de enfermedad para estos pacientes fue: renal en el $55,55 \%(n=5)$ de los casos, neurológica en el $33,33 \%(n=3)$ de los casos y dermatológica en el

\section{Correspondencia: \\ Yaiza Martínez Delgado \\ Servicio de Nefrología. Parc de Salut Mar \\ Passeig Maritim, 25-29. 08003 Barcelona. España \\ Email: yaimardel@gmail.com}

$11,11 \%(n=1)$. De las 93 sesiones totales hubo problemas relacionados con la canalización de un acceso vascular periférico en el $52,69 \%$ de las sesiones $(n=49)$.

Conclusiones: Debido a que en más de la mitad de las sesiones se presentaron complicaciones en el abordaje del acceso vascular periférico, se concluye que es necesario el estudio vascular ambulatorio del paciente antes de la primera sesión, pudiendo ser útil el uso de la punción ecoguiada.

PALABRAS CLAVE: recambio plasmático terapéutico; plasmacentrifugación; acceso vascular periférico.

\section{Analysis of the use and complications of peripheral vascular access in centrifuge-based therapeutic plasma exchange}

\section{Abstract}

Introduction: Therapeutic plasma exchange is an extracorporeal blood purification technique. Centrifuge-based therapeutic plasma exchange (plasmacentrifugation) requires flows of $50 \mathrm{ml} / \mathrm{min}$ which allows the exchange with peripheral vascular accesses. The main objective of this study is to analyze the use and complications of peripheral vascular access in plasmacentrifugation sessions.

Material and Method: Descriptive observational longitudinal study on the analysis of peripheral vascular access complications in plasmacentrifugtion sessions between January 2017 and March 2018. 
Results: A total of 93 plasmacentrifugation sessions were included. Sessions were carried out in 9 patients with a median of 7 [P25:6; P75:10.5] per patient. $66.66 \%(n=6)$ were women. Centrifugation was used in $89.24 \% \quad(n=83)$ of the sessions and in $10.75 \%$ $(n=10)$ photoaferesis. The etiologies of the disease for these patients were: renal in $55.55 \% \quad(n=5)$ of cases, neurological in $33.33 \%(n=3)$ of cases and dermatological in $11.11 \%(n=1)$. There were problems related to the channeling of peripheral vascular access in $52.69 \%$ of the sessions $(n=49)$.

Conclusions: Because in more than half of the sessions there were complications in the channeling of peripheral vascular access, it is concluded that ambulatory vascular study of the patient is necessary before the first session, the use of ultrasound guided puncture may be useful.

KEYWORDS: therapeutic plasma exchange; plasmacentrifugation; peripheral vascular access.

\section{Introducción}

El recambio plasmático terapéutico (RPT) es una técnica extracorpórea de depuración de la sangre, mediante la cual se elimina el plasma con el objetivo de eliminar aquellas sustancias patogénicas capaces de generar o perpetuar una enfermedad. En esta técnica, se realiza la extracción de un volumen variable de plasma del paciente que es sustituido por soluciones de reposición (plasma fresco cuarentenado, seroalbúmina al $5 \%$ o coloides) que mantengan el volumen y la presión oncótica ${ }^{1}$. Esta técnica se ha integrado en las unidades de nefrología, ya que precisa de un personal entrenado y familiarizado con el manejo tanto de los circuitos extracorpóreos cómo del acceso vascular y las posibles complicaciones que se puedan derivar de $a^{a m b o s^{2}}$.

En los años 80 se fundó la American Society for Apheresis (ASFA) y se crearon las guías para el uso del RPT. Estas guías se publican de forma periódica y se realizan revisiones sistemáticas acerca de las indicaciones terapéuticas para esta técnica. La evidencia de las guías se divide en 4 grados de recomendación según la eficacia y la calidad de las investigaciones. Actualmente se tratan más de 80 enfermedades de diferentes especialidades médicas ${ }^{3}$. A la misma vez, los avances tecnológicos que se han evidenciado en los equipos de aféresis automatizados han hecho que estos procedi- mientos cada vez sean más seguros, rápidos y efectivos. Actualmente, las técnicas principales de RPT son la separación por centrifugación y la separación por filtración por membrana. Estas técnicas permiten la separación exclusiva del componente deseado según la densidad, el tamaño y el peso molecular, por lo que se reduce la pérdida no deseada de otras proteínas y componentes del plasma ${ }^{4}$.

Los sistemas por centrifugación separan los elementos celulares del plasma por medio de elementos giratorios que dividen los distintos componentes según su peso molecular mediante fuerza centrífuga. Los separadores celulares más actuales son de flujo continuo, en los que la sangre del paciente penetra en la máquina, es centrifugada separando el componente deseado y el remanente se retorna a un flujo constante sin interrupción. Este procedimiento se realiza mediante 2 accesos venosos, utilizándose a modo de salida y entrada de la sangre respectivamente. Las máquinas centrífugas pueden comprimir los glóbulos rojos a un hematocrito del $80 \%$, por lo que pueden eliminar el $80 \%$ o más del plasma que reciben. Así, para eliminar 1,2 veces el volumen plasmático del paciente, necesitan procesar solo 1,5 veces el volumen de sangre, precisando un flujo bajo, que permite la utilización de vías venosas periféricas ${ }^{5}$.

El acceso vascular para los RPT se ha de elegir en función del tipo de procedimiento prescrito, el mapeo vascular del paciente, la frecuencia, duración y enfermedad de base del paciente. Las diferentes posibilidades incluyen: fístula arteriovenosa nativa o Protésica (FAVn o FAVp), catéteres venosos centrales tunelizados o temporales, o catéteres periféricos ${ }^{6}$.

En el caso de RPT por centrifugación, el bajo flujo sanguíneo que precisa nos permite la utilización de vías periféricas evitando así los riesgos que acompañan a un catéter venoso central (CVC) (infección, trombosis, disfunción, estenosis, etc.). Puesto que no todas las venas periféricas se pueden localizar ni visualmente ni por palpación, con la colocación del acceso venoso periférico guiado por ultrasonido, se beneficia a los pacientes ya que se disminuye el número de intentos fallidos, evitando posibles complicaciones (extravasación, hematomas, punciones fallidas, etc.) que comprometen el árbol vascular y dificultando aún más la colocación de nuevos accesos. Además, se reduce la necesidad y el gasto adicional que supone la colocación de $\mathrm{CVC}^{7}$. A pesar de estos beneficios reportados utilizando el ecógrafo para la inserción de catéteres 
periféricos, en nuestro entorno aún no se han instaurado estas rutinas de manera sistemática y enfermería sigue utilizando la palpación para su colocación. Debido a estos aportes de la bibliografía, nos planteamos analizar el uso y complicaciones del acceso vascular periférico en las sesiones de plasmacentrifugación que se realizaron en el servicio de nefrología en Hospital del Mar de Barcelona.

\section{Material y Método}

Se realizó un estudio observacional, descriptivo y longitudinal en el servicio de Nefrología del Hospital del Mar de Barcelona en el periodo comprendido entre enero de 2017 y marzo de 2018. Se evaluaron 93 sesiones de RPT mediante centrifugación en 9 pacientes. Como criterios de inclusión se estipuló ser mayor de 18 años en tratamiento de RPT por centrifugación y dar su consentimiento para poder revisar la historia clínica personal para analizar los datos. Como criterios de exclusión se identificó la negativa a participar voluntariamente cediendo los datos personales para su análisis o presentar un estado crítico o algún tipo de barrera en la comunicación que impidiera el correcto entendimiento del estudio. Se analizaron las complicaciones relacionadas con el acceso vascular periférico en las sesiones de RPT por plasmacentrifugación realizadas a los pacientes de nuestro centro. Se recogieron las variables sociodemográficas y clínicas de los pacientes (sexo, edad, patología de base, índice de masa corporal e índice de comorbilidad de charlson) y se recogieron variables de las sesiones (número de sesiones realizadas, tipo de técnica realizada, acceso vascular utilizado, complicaciones en la colocación de las vías y la imposibilidad de realización de la técnica debido a la falta de obtención de acceso vascular). Para la realización del procedimiento se utilizó la maquina Spectra Optia - Terumo BCT. El anticoagulante empleado en todas las sesiones fue el citrato (ACD-A). Se revisaron las historias clínicas informatizadas de los pacientes y los registros específicos de enfermería para recoger las variables a estudio. Se realizó un análisis descriptivo de las variables sociodemográficas con porcentajes y frecuencias para las variables cualitativas y media y desviación estándar para las cuantitativas en caso de seguir una distribución normal, o bien mediana y rango intercuartílico en caso contrario.

Este estudio se basa en los principios básicos que guían la atención y la investigación en el campo sociosanitario: principio de autonomía y respeto a las personas, principio de beneficencia, principio de justicia y principio de no maleficencia, incluido en el Código de ética de enfermería en Cataluña, la Declaración de Helsinki, el Reglamento Europeo 2016/679 de Protección de Datos y en la Ley Orgánica de Protección de Datos y Garantía de los Derechos Digitales de España. No se introdujo ningún dato que permita la identificación de ningún paciente respetando así la Ley de Protección de datos de Carácter Personal para garantizar que la base de datos no se podrá utilizar de forma errónea. La explicación verbal y escrita sobre el estudio fue dada por las enfermeras responsables del estudio y el consentimiento informado se obtuvo de la misma manera.

\section{Resultados}

Se evaluaron un total de 93 sesiones, con una mediana de 7 (P25:6; P75:10,5) sesiones por paciente. La edad media de los pacientes fue de 50,66 $\pm 15,34$ años. El $66,66 \%(n=6)$ fueron mujeres.

La etiología de base prevalente fue la renal en un $55,55 \%(n=5)$, seguida de la neurológica en un $33,33 \%(n=3)$ y la dermatológica en un $11,11 \%$ $(n=1)$. En la Tabla 1 se puede observar las principales causas etiológicas dentro de cada una de estas categorías.

Tabla 1. Características sociodemográficas y clínicas de la muestra.

\begin{tabular}{ll}
\hline Edad (media; DE) & $50,66(15,34)$ años \\
\hline Sexo (\%; $\mathbf{n})$ & $33,33 \%(3)$ \\
Hombres & $66,66 \%(6)$ \\
Mujeres & \\
\hline Etiología de enfermedad (\%; $\mathbf{n}$ & $55,55 \%(5)$ \\
$\quad$ Renal & $11,11 \%(1)$ \\
$\quad$ Vasculitis reno pulmonar & $33,33 \%(3)$ \\
$\quad$ Rechazo agudo TR & $11,11 \%(1)$ \\
$\quad$ Hialinosis segmentaria y focal & $33,33 \%(3)$ \\
$\quad$ Neurológica & $22,22 \%(2)$ \\
$\quad$ Miastenia gravis & $11,11 \%(1)$ \\
$\quad$ Esclerosis multiple & \\
& $11,11 \%(1)$ \\
\hline
\end{tabular}

DE: Desviación estandar;TR: Trasplante renal; Sd: Síndrome.

Del número total de sesiones a los que se le realizó RPT, en un $89,24 \%(n=83)$ de estas se usó la plasmacentrifugación y en un $10,75 \%(n=10)$ la fotoaféresis. Se analizó el índice de comorbilidad de Charlson de los 
pacientes, con una mediana de $4\left(P_{25} 2 ; P_{75} 4\right)$ lo que se corresponde con una comorbilidad alta. Al analizar el IMC, encontramos que un $44,44 \%(n=4)$ tenían sobrepeso, un $44,44 \%(n=4)$ peso normal y el $11,11 \%$ $(n=1)$ restante presentaba delgadez aceptable.

De las 93 sesiones totales se encontraron complicaciones para la canalización de un acceso vascular periférico en el $52,69 \%$ de las sesiones ( $n=49)$ (Tabla 2). En 4 sesiones se requirió la colocación de un catéter venoso medial por parte de la enfermera referente del hospital. En 7 sesiones, fue imposible la canalización del acceso vascular periférico y la colocación de un acceso venoso medial, precisándose de la colocación de un catéter venoso central pediátrico para hemodiálisis por parte del nefrólogo responsable. En las 38 sesiones restantes, enfermería requirió más de 2 punciones para la obtención de un acceso vascular adecuado.

Tabla 2. Número y porcentaje de incidencias relacionadas con la canalización de un Acceso Vascular Periférico (AVP).

\begin{tabular}{ll}
\hline Sin incidencias $(\mathrm{n} ; \%)$ & $44(47,31 \%)$ \\
\hline Con incidencias $(\mathrm{n} ; \%)$ & $49(52.69 \%)$ \\
& Imposibilidad de canalizar AVP: \\
& $\begin{array}{l}\text { Més de } 2 \text { punciones para canalizar } \\
\text { un AVP: } 38(40,86 \%)\end{array}$ \\
\hline
\end{tabular}

Los recambios terapéuticos fueron bien tolerados en todos los pacientes y no hubo complicaciones relacionadas con el procedimiento. Pese a las complicaciones con el acceso vascular, no se imposibilitó ninguna sesión.

\section{Discusión}

En el presente estudio se pretende analizar el uso y las complicaciones del acceso vascular periférico en los RPT por centrifugación. Según los resultados presentados en este estudio, tal y como también indica la bibliografía referente al tema, el acceso vascular de elección para la plasmacentrifugación es el periférico por las ventajas ya mencionadas, pero dada la alta tasa de complicaciones relacionadas con su abordaje, como también referencian Ipe y Marques en su artículo ${ }^{8}$, usar técnicas ecográficas para la canalización de vías periféricas es una solución para pacientes donde no se palpan fácilmente, pero requiere un entrenamiento específico para disminuir el número de intentos fallidos.
Según Rodríguez-Calero y Blanco-Mavillard, la dificultad en la canalización venosa periférica es un problema cotidiano en la práctica clínica, entre un 10 y un $24 \%$ de los adultos pueden presentarlo siendo problema cínico y asistencial ${ }^{10}$. Existe la clasificación de "Vía Venosa Difícil", cuya definición más extendida es aquella en la que se realizan 20 más punciones sin éxito, o VVP en las que es preciso utilizar técnicas de apoyo como la ecografía o la necesidad de colocar un $\mathrm{CVC}^{11}$. Vinculando las variables asociadas con la dificultad de canalización con los resultados obtenidos en nuestro estudio, observamos que nuestros pacientes no presentaban situaciones agudas como hipotensión arterial o deshidratación ni inestabilidad hemodinámica, no tenían antecedentes de abuso de drogas por vía parenteral, pero sí unas condiciones de cronicidad que se corresponden con el alto índice de comorbilidad CCI obtenido. La obesidad también se describe como una condición de cronicidad junto con la pluripatología crónica avanzada ya que conlleva contactos repetidos con el sistema sanitario especializado, factor considerado como potenciador del "agotamiento vascular"12.

El acceso vascular es el medio de elección a través del cual se realiza el RPT, por lo tanto, es dónde reside en gran parte el éxito de la técnica ${ }^{13}$. Sin un flujo adecuado se puede realizar el procedimiento, pero de manera subóptima, alargando el tiempo, disminuyendo la eficiencia o incluso necesitando interrumpir la técnica. La elección del acceso variará en función de la urgencia del procedimiento, tiempo real, volumen de plasma a intercambiar, frecuencia y duración (días o meses), y de las condiciones del paciente: anatomía vascular, estado hemodinámico, hidratación, uso de medicación, etc. Debe resistir una presión negativa alta sin colapsarse y al mismo tiempo, estar en un vaso sanguíneo grande capaz de tolerar una presión positiva alta de retorno. Por esto mismo, la elección del catéter debe ser de un calibre 18 o superior. Tal y como cita Golestaneh ${ }^{6}$ las vías periféricas presentan ventajas con respecto al resto de accesos vasculares utilizados (CVC, FAVn, FAVp) como son la baja tasa de infecciones y la inmediatez de su uso, y desventajas como los hematomas, daño de nervios y esclerosis de las venas, además del disconfort del paciente. En una revisión de 2007 del uso del acceso vascular en aféresis en todo el mundo, indica que existe una variación de la practica en los principales métodos utilizados siendo el acceso venoso periférico el método de acceso más utilizado en Europa y Australia, mientras que los CVC lo son a su vez en América del Norte, centro y sur y en Asia ${ }^{14}$. 
De esta manera, la bibliografía recomienda el uso del acceso vascular periférico para la realización de la técnica de plasmacentrifugación y existen también evidencias de que el uso del ecógrafo es la mejor opción para su colocación ${ }^{15}$. Dado que el uso de este instrumento no está extendido en nuestro entorno, validamos impulsar programas de formación para integrarlo en la práctica clínica diaria presentando el alto índice de complicaciones asociadas a la colocación de AVP mediante palpación y visualización exclusivamente. Las contraindicaciones absolutas para la realización de los RPT son escasas, pero son fundamentalmente dos; la inestabilidad hemodinámica del paciente y la falta de un acceso vascular adecuado para su realización. Estas estrategias nos permitirán reducir la prevalencia de complicaciones y la preservación del capital venoso del paciente.

Las principales limitaciones del estudio han sido el tamaño de la muestra y su representatividad. Pese a esto, se ha logrado validar las aportaciones que ya ha realizado la bibliografía anteriormente y esto permitirá mejorar los planes de cuidados en nuestro entorno concreto.

Debido a la alta tasa de complicaciones encontrada en cuanto al abordaje de los accesos vasculares periféricos, para la técnica de recambios plasmático terapéutico, en nuestro servicio, consideramos que es necesario utilizar estrategias más eficientes como el estudio vascular ambulatorio del paciente antes de la primera sesión, la valoración conjunta por el equipo multidisciplinar que lo atenderá y la implantación de protocolos de punción ecoguiada o asistida de forma temprana en los pacientes que así lo precisen detectando precozmente pacientes de alto riesgo, evitando la multipunción o el uso de catéteres de acceso central.

\section{Conflicto de intereses}

Los autores declaran que no existe ningún conflicto de interés.

\section{Financiación}

Este estudio no ha sido financiado.

\section{Recibido: 07-12-18 \\ Revisado: 01-02-19 \\ Modificado: 15-04-19 \\ Aceptado: 28-05-19}

\section{Bibliografía}

1. Madore F, Lazarus JM, Brady HR. Therapeutic Plasma Exchange in Renal Diseases. J. Am. Soc. Nephrol.1996;7:367-86.

2. Anaya F. Por qué la aféresis terapéutica debe pertenecer a la especialidad de nefrología. Rev Nefrología.2011;31(4):379-81.

3. Aznar Barbero S, Bel Cegarra R, Chica Arellano J. Resultados de intercambio plasmático en síndrome del hombre rígido: experiencia de un caso. Rev Enfermería Nefrológica.2014;17(3):216-21.

4. Okafor C, Ward DM, Mokrzycki MH, Weinstein R, Clark $\mathrm{P}$, Balogun RA. Introduction and overview of therapeutic apheresis. J Clin Apher.2010;25:240-9.

5. Guillermo J, Pons E, Serrano R, Lozano M, Cid J, Cervera R, Espinosa G. Recambio plasmático en las enfermedades autoinmunes sistémicas. Sem Fund Esp Reumatol.2013;2(14):43-50.

6. Golestaneh L, Mokrzycki MH.Vascular access in therapeutic apheresis: J Clin Apher.2013;28(1):64-72.

7. Fortun J. Infecciones asociadas a dispositivos intravasculares utilizados para la terapia de infusión. Enferm Infecc Microbiol Clin.2008;3(26):168-74.

8. Ipe $T$, Marques M. Vascular access for therapeutic plasma exchange. Transfusion.2018;1:580-9.

9. Rodriguez-Calero MA, Blanco-Mavillard I. Dificultad en la canalización de vías periféricas: ¿existen factores de riesgo?. Evidentia. 2017;14,(1). Disponible en https://dialnet.unirioja.es/servlet/articulo?codigo $=6376673$.

10. Sabri A, Szalas J, Holmes KS, Labib L, Mussivand T. Failed attempts and improvement strategies in peripheral intravenous catheterization. Biomed Mater 
Eng. 2013;23(1-2):93-108. [Consultado 5 diciembre 2018]. Disponible en: http://www.ncbi.nlm.nih. gov/pubmed/23442240.

11. Egan G, Healy D, O'Neill H, Clarke-Moloney $M$, Grace $\mathrm{P}$ a, Walsh SR. Ultrasound guidance for difficult peripheral venous access: systematic review and meta-analysis. Emerg Med J. 2015;30(7):5216. [Consultado 5 diciembre 2018]. Disponible en: http://www.ncbi.nlm.nih.gov/pubmed/22886890.

12. Juvin P, Blarel A, Bruno F, Desmonts J-M. Is peripheral line placement more difficult in obese than in lean patients? Anesth Analg. 2003;96:1218, table of contents.

13. Shaz BH, Schwart J and Winters JL. How we developed and use the American Society for Apheresis guidlines for therapeutic apheresis procedure. Transfusion. 2014;54:17-25.

14. Malchesky PS, Koo AP, Skibinski CI, Hadsell AT, Rybicki LA. Apheresis technologies and clinical applications: the 2007 international apheresis registry. Ther Apher Dial. 2010;14(1):52-73.

15. Hanafusa N, Noiri E, Nangaku M. Vascular access puncture under ultrasound guidance. Ther Apher Dial. 2014;18(2):213-4.

Este artículo se distribuye bajo una Licencia Creative Commons Atribución-NoComercial 4.0 Internacional. https://creativecommons.org/licenses/by-nc/4.0/

\section{Open Access C) (i) (8)}

